\title{
Cardiovascular disease in patients with chronic kidney disease: a neglected subgroup
}

\author{
Arun Kumar Subbiah, Yogesh K Chhabra, Sandeep Mahajan
}

Department of Nephrology, All India Institute of Medical Sciences, New Delhi, India

\section{Correspondence to}

Dr Sandeep Mahajan,

Department of Nephrology, All India Institute of Medical Sciences, New Delhi 110029, India; mahajansn@yahoo.com

Received 18 July 2016 Revised 10 October 2016 Accepted 13 October 2016

\section{CrossMark}

To cite: Subbiah AK,
Chhabra YK, Mahajan S.
Heart Asia 2016:8:56-61.
doi:10.1136/heartasia-2016-
010809

\section{ABSTRACT}

The burden of non-communicable diseases has increased exponentially over the past decade and they account for majority of the health-related morbidity and mortality worldwide. In line with this, the prevalence of chronic kidney disease (CKD) has been increasing over the years. CKD progresses through stages and it is well known that patients are more likely to die than to progress to end-stage renal disease. The presence of multiple classical and novel risk factors predisposes this group of patients to premature cardiovascular mortality. Though being a common entity, prevention, diagnosis and treatment of cardiovascular diseases in CKD are mired with controversies. This is due to the fact that many of the well-established diagnostic modalities and treatment strategies have not been studied in detail in patients with CKD. Moreover, most of the studies have excluded patients with renal dysfunction though they are at a higher risk for adverse outcomes and require specific dose modifications. This has limited the evidence base for optimal decision making. In this review, we aim to cover the risk factors, diagnosis and effectiveness of interventional strategies in patients with CKD.

\section{INTRODUCTION}

Renal origin of cardiovascular disease (CVD) was first suggested by Richard Bright as early as in 1836 . This has been confirmed by multiple epidemiological studies wherein as compared with the general population, patients with chronic kidney disease (CKD) had more frequent and severe CVD. CKD per se is considered to be a coronary artery disease (CAD) equivalent and in fact persons with early stages of CKD are more likely to die of CVDs than progress to end-stage renal disease (ESRD). ${ }^{1}$ Though the magnitude of this risk has been repeatedly discussed, the trials have conveniently excluded patients with renal impairment. Thus, evidencebased management of CVD in CKD is lacking. This has significant treatment implications, as measures directed at preventing the progression of CKD would prevent cardiovascular morbidity and mortality too. The concept that CVD and CKD can initiate and perpetuate one another led to the creation of cardiorenal syndrome as a separate clinical entity. ${ }^{2}$ The mechanism underlying the increased risk of cardiovascular events in patients with CKD has not been well defined. Several factors are believed to be responsible for such an association. Even after adjusting for the coexisting multiple risk factors, CKD appears to be the major factor in determining cardiovascular morbidity and mortality. ${ }^{3}$ In this review, we present the available evidence on the CVD burden in CKD, risk factors, diagnostic modalities and treatment options with future research questions on CVD in CKD.

\section{CVD BURDEN-EPIDEMIOLOGY}

The spectrum of CVD in CKD includes ischaemic heart disease, congestive heart failure, arrhythmias and peripheral vascular disease. For our discussion we shall focus on ischaemic heart disease as this accounts for the major chunk of the cases and also cardiovascular morbidity and mortality. From data available from several epidemiological studies, it is obvious that patients with CKD are more prone to develop CVD. ${ }^{4}$ CVD kills patients with CKD more frequently and prematurely. Initially, it was thought that this is limited to the ESRD population who were 20-30 times more likely to die of CVDs in comparison with the general population. This increased risk, however, is spread over the entire spectrum of CKD across all stages of CKD. The largest population-based study by Go et al involving 1120295 adults revealed that adjusted HR for cardiovascular events increased inversely with the estimated glomerular filtration rate (eGFR); 1.4 for eGFR of $45-59 \mathrm{~mL} / \mathrm{min}$ per $1.73 \mathrm{~m}^{2}, 2.0$ for eGFR of $30-44 \mathrm{~mL} / \mathrm{min}$ per $1.73 \mathrm{~m}^{2}, 2.8$ for eGFR of $15-$ $29 \mathrm{~mL} / \mathrm{min}$ per $1.73 \mathrm{~m}^{2}$ and 3.4 for eGFR $<15 \mathrm{~mL} /$ min per $1.73 \mathrm{~m}^{2}$, respectively. The adjusted risk of hospitalisation and mortality followed a similar pattern. A collaborative meta-analysis of 10 cohorts with 266975 patients by van der Velde et $a l^{6}$ revealed similar trend of cardiovascular mortality with eGFR. In the same study, albuminuria was also associated with the risk of all-cause mortality. In the Hypertension Detection and Follow-up Program, a linear relation was found between serum creatinine levels and cardiovascular mortality, with a fivefold difference between the lowest and highest serum creatinine strata. Similar results were obtained in the Hypertension Optimal Treatment study. The United States Renal Data System (USRDS) 2014 annual report reinforces this fact wherein it is stated that the prevalence of any CVD is double in patients with CKD $(69.8 \%$ vs $34.8 \%)$. Manjunath $e t \mathrm{al}^{7}$ quantified the risk of CVD and showed that for every $10 \mathrm{~mL} / \mathrm{min}$ per $1.73 \mathrm{~m}^{2}$ reduction in eGFR, the risk for cardiovascular mortality increased by $5 \%$.

The risk of CVD in CKD varies with the degree of renal impairment and proteinuria, and depends on the rate at which these changes occur. In the Multiple Risk Factor Intervention Trial, the risk for CVD was high among those who had worsening renal dysfunction during the course of the study. Coexisting proteinuria increases the propensity to develop CVD and rapid deterioration in renal function adds to the risk. Irie $e t a l^{8}$ also confirmed the independent yet additive effects of proteinuria and eGFR on the risk of cardiovascular mortality in a cohort of Japanese patients. 
CVD in turn also enhances the rapidity of progression of CKD as is seen in the Chronic Renal Insufficiency Cohort (CRIC) study. ${ }^{9}$ In the CRIC study, a history of heart failure was independently associated with a 29\% higher risk for declining renal function or ESRD. Also in the combined analyses of the Atherosclerosis Risk in Communities Study and Cardiovascular health study, it was shown that participants with CVD at baseline were more likely to experience decline in renal function. ${ }^{10}$

\section{RISK FACTORS FOR CVD IN PATIENTS WITH CKD}

The increased incidence of CVD in CKD is only partially accounted for by the higher prevalence of traditional risk factors in these patients. This has turned our attention on the non-traditional or the 'novel' risk factors unique to CKD.

The traditional risk factors for CVD such as increasing age, hypertension, dyslipidaemia, diabetes, smoking and obesity are risk factors for CKD as well and hence are common in patients with CKD. The non-traditional or 'novel' risk factors are 'uraemia specific', or at least much more common in patients with CKD than in the general population. These include albuminuria, anaemia, hyperparathyroidism, metabolic bone disease, hyperhomocysteinaemia, malnutrition, apolipoprotein isoforms, inflammation, endothelial dysfunction and oxidative stress. The various risk factors traditional and non-traditional tend to have an additive effect and hasten atherosclerosis and progression of CKD. ${ }^{11}$ This complex relationship between traditional and nontraditional risk factors and CVD and CKD is depicted in figure 1.

Heart Outcomes Prevention Evaluation (HOPE), Losartan Intervention for Endpoint Reduction in Hypertension (LIFE) and Prevention of Renal and Vascular Endstage Disease (PREVEND) studies have shown unequivocally that albuminuria plays a significant role in the pathogenesis of CVD. ${ }^{12}$ Anaemia leads to hyperdynamic circulation and generalised vasodilatation, which acts as a stimulus for neurohormonal activation and salt and water retention. Anaemia of CKD increases the risk of cardiovascular morbidity and mortality by causing left ventricular (LV) systolic dysfunction and left ventricular hypertrophy $(\mathrm{LVH})$. Prior studies in patients on dialysis have shown that LVH is a reliable predictor of morbidity and mortality. Weiner $e t a l^{13}$ analysed 2423 patients with CKD and concluded that the presence of both anaemia and LVH conferred a fourfold higher risk for the composite outcome of myocardial infarction or death, while the presence of either of them had a threefold higher risk in comparison with patients with CKD without either of these

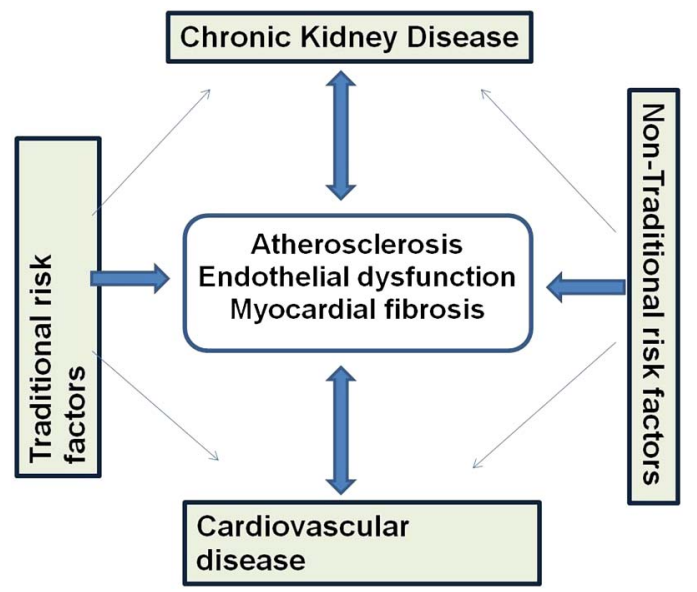

Figure 1 Interrelationship between traditional and non-traditional risk factors and cardiovascular disease and chronic kidney disease. risk factors. C-Reactive protein (CRP) and interleukin-6 are the most commonly measured inflammatory markers. Clinical utility of measuring CRP is limited as even though it had shown to be predictive of cardiovascular mortality in the Modification of Diet in Renal Disease (MDRD) study, it was not found to be useful in the Irbesartan in Diabetic Nephropathy trial. The studies involving inflammatory markers are mostly observational and until large-scale trials with sufficient number of patients and adequate follow-up are available it would not be possible to comment on their utility. Asymmetric dimethylarginine (ADMA) inhibits endogenous nitric oxide synthase (NOS) resulting in endothelial dysfunction. Levels of ADMA increase with worsening renal dysfunction, and studies have shown a positive correlation between levels of ADMA with cardiovascular mortality. Role of L-arginine, which competitively inhibits ADMA, antioxidants such as acetylcysteine and vitamin E, needs further studies before being recommended to counteract the oxidative stress in patients with CKD. Abnormal bone mineral metabolism with elevated calcium phosphorous product leads to vascular calcification that adversely affects the cardiovascular system. Presently, the phosphaturic hormone FGF23 has been found to be associated with $\mathrm{LVH}$ and vascular calcification. Numerous reports have linked elevated FGF23 to progression of ESRD, CVD and death. Apart from being a highly sensitive biomarker of toxicity due to phosphate, FGF23 exhibits a direct end organ toxic effect on the heart. Several cross-sectional studies across the entire spectrum of varying degrees of renal dysfunction have demonstrated that elevated FGF23 levels correlate with higher LV mass index and LVH, which in turn predispose the patients to congestive heart failure and arrhythmias. ${ }^{14}$ This was also seen in The Homocysteine in Kidney and End Stage Renal Disease (HOST) study where high FGF23 levels superseded all known classic cardiovascular risk factors and increased the risk of myocardial infarction, initiation of maintenance haemodialysis and all-cause mortality. Higher FGF23 results in impaired vasoreactivity, endothelial dysfunction and increased arterial stiffness. Though it has been nearly a decade since the landmark paper by Gutiérrez et $a l^{15}$ demonstrating FGF23 to be independently associated with mortality in incident haemodialysis patients, research is still underway to definitively establish a causal link to CVD. A potential benefit of therapies targeting the FGF23Klotho axis needs to be evaluated.

\section{DIAGNOSIS OF CVD IN CKD}

The classic triad of ischaemic symptoms, elevated cardiac biomarkers and ECG changes is frequently absent in patients with $\mathrm{CKD}$, making diagnosis of CAD challenging. Moreover, there is paucity of data on the utility of various diagnostic modalities in patients with CKD. This is due to the exclusion of patients with renal dysfunction from major randomised trials and in part due to reduced negative predictive value of these tests secondary to the increased prevalence of CAD in this population. ${ }^{16}$

Structural and functional cardiac abnormalities are common in patients with CKD. The most widely available investigation in resource-limited setting is ECG. Abnormal ECGs are common and in one study $46 \%$ of patients had some abnormality thereby reducing the specificity for diagnosis of CAD. ${ }^{17}$ ECGs are useful to detect $\mathrm{LVH}$ and bundle branch blocks. Two-dimensional (2D) transthoracic echocardiography is easily available, inexpensive but is operator dependent and is generally used to measure LV mass index and see regional wall motion abnormalities suggestive of CAD. Exercise testing is of low utility due to low exercise tolerance in patients with CKD. Cardiac MRI is particularly useful to detect myocardial scarring. Stress cardiac magnetic resonance 
is useful for detecting coronary artery stenosis $\geq 70 \%$, with a reported sensitivity of $100 \%$ and a specificity of $90 \%$ but issues of cost, availability, use in individuals with GFR $<30 \mathrm{~mL} / \mathrm{min}$ per $1.73 \mathrm{~m}^{2}$ limits its use. CT angiography has good sensitivity and specificity in normal individuals. Utility of computed tomography angiography (CTA) in CKD is limited because of the presence of significant coronary calcification in this population. Though some studies have shown promising results, they have not compared CTA with the 'gold standard' conventional angiography. Single-photon emission CT scans have lower sensitivity to detect CAD in patients with CKD as higher baseline adenosine causes vasodilation and interfere with the test. Moreover, antianginals used in patients with CKD reduce the ischaemic burden and reduce sensitivity. However, they have excellent negative predictive value as shown in the study by Patel et al. ${ }^{18}$ Dobutamine stress echocardiography (DSE) is useful for prognostification in patients with CAD. The presence of LVH reduces the sensitivity of DSE. Submaximal heart rate due to limited exercise tolerance adds to the problem. Studies by De Lima $e t a l^{19}$ and Reis $e t a l^{20}$ showed a poor sensitivity and positive predictive value (PPV) for DSE for the diagnosis of CAD. Stress thallium scintigraphy is more sensitive but less specific than DSE. In the study by Vandenberg et al, ${ }^{21}$ stress thallium scan had $62 \%$ sensitivity and $76 \%$ specificity for CA stenosis of $>75 \%$ in comparison with conventional angiography. The utility of myocardial perfusion studies in identifying patients with CKD with future risk of CAD was shown in the meta-analysis by Rabbat et al. ${ }^{22}$ With regard to using non-invasive tests based on comorbid illnesses, myocardial perfusion scintigraphy (MPS) is better in patients with uncontrolled hypertension and arrhythmia, while DSE should be used in patients with reversible airways disease and hypotension. The recent review by Wang et $\mathrm{al}^{23}$ on prognostic value of MPS, DSE or coronary angiography in potential kidney transplant recipients showed that in the preoperative risk assessment, the non-invasive tests (MPS and DSE) are 'at least as good' as an abnormal coronary angiography to predict major cardiovascular events and mortality. Whether this conclusion is applicable in the normal scenario (as against the perioperative evaluation) is a matter of concern and the choice of screening method would depend on risk stratification as explained below. Table 1 shows the utility of various diagnostic modalities for the diagnosis of CVD.

Table 1 Utility of various diagnostic modalities for diagnosis of cardiovascular disease in CKD

\begin{tabular}{ll}
\hline Diagnostic modality & Comment \\
\hline ECG & $\begin{array}{l}\text { Should be done yearly; for LVH and baseline } \\
\text { rhythm } \\
\text { LV function, RWMA, valvular disease; operator } \\
\text { dependent } \\
\text { Vesting echocardiographe sensitivity; effect of antihypertensive } \\
\text { agents; good negative predictive value }\end{array}$ \\
Cardiac SPECT & $\begin{array}{l}\text { More specific than SPECT for CAD; LVH is } \\
\text { confounder; exercise intolerance }\end{array}$ \\
$\begin{array}{l}\text { Dobutamine stress } \\
\text { echocardiography }\end{array}$ & $\begin{array}{l}\text { Good sensitivity and specificity; not in eGFR } \\
\text { Stress CMR }\end{array}$ \\
Coronary CT angiography & $\begin{array}{l}\text { Not in established CAD/ESRD; higher burden of } \\
\text { coronary calcium confounder } \\
\text { Gold standard; invasive, loss of residual renal } \\
\text { function }\end{array}$ \\
\hline
\end{tabular}

CAD, coronary artery disease; CMR, cardiac magnetic resonance; ESRD, end-stage renal disease; $L V$, left ventricle; LVH, left ventricular hypertrophy; RWMA, regional wall motion abnormalities; SPECT, single-photon emission CT.
American Heart Association/American College of Cardiology Foundation (AHA/ACCF) recommends that non-invasive stress testing may be considered in kidney transplantation candidates with no active cardiac conditions on the basis of the presence of multiple CAD risk factors regardless of functional status. ${ }^{24}$ The suboptimal performance of current non-invasive tests makes coronary angiography the desirable investigation in high-risk patients. High-risk patients are clinically defined by clinical evidence of actual or past vascular arterial disease, heart failure and previous stroke or myocardial infarction.

Serum biomarkers routinely used in non-CKD population are less useful as they are elevated without evidence of myocardial necrosis. This increase is not secondary to reduced renal clearance as thought earlier but due to silent micro infarction and myocardial apoptosis. ${ }^{25}$ With respect to cardiac troponins (cTn), though it was initially believed that cTn-I is more reliable than cTN-T, none of the guidelines recommend one troponin over another. Moreover with the availability of newer more sensitive assays for cTn-I, it is no longer more specific in CKD population. In the meta-analysis with a total of 14 studies, the sensitivity and specificity for the diagnosis of acute coronary syndrome (ACS) ranged from $71 \%$ to $100 \%$ and $31 \%$ to $86 \%$, respectively, for cTn-T, and from $43 \%$ to $94 \%$ and $48 \%$ to $100 \%$, respectively, for $\mathrm{cTn}-\mathrm{II}^{26}$ The general consensus is that serial values rather than a single value should be considered for diagnosis of acute myocardial infarction in patients with CKD. Even when serial values are used, the timing of haemodialysis should be taken into consideration as levels vary with the duration of dialysis and use of high flux membranes. Promising results have been obtained when cTn are used to assess prognosis in patients with CKD. Published meta-analysis revealed that elevated cTn-T was associated with a threefold increase in all-cause mortality and cTn-I was associated with a 2.7-fold increase in all-cause mortality. In the Global Use of Strategies to Open Occluded Coronary Arteries IV trial with 7033 patients with suspected ACS, an elevated cTn-T was strongly predictive of poor shortterm prognosis regardless of the degree of renal dysfunction. Due to lower sensitivity of creatine kinase $\mathrm{MB}$ isoenzyme (CK-MB), troponin assay is the preferred biomarker. The use of $\mathrm{CRP}$ as an added biomarker for prognostification needs further study. B-type natriuretic peptide and NT-pro-B-type natriuretic peptide (BNP) also add prognostic value in ACS. Though their levels are elevated in renal failure, studies have shown that increased levels correlate with LV wall stress and have prognostic utility for mortality. ${ }^{27}$

\section{MANAGEMENT OF CVD IN CKD}

The mainstay of medical management of CVD is therapy with aspirin, statins, ACE inhibitors (ACEi) or angiotensin receptor blockers (ARBs) and $\beta$ blockers. Optimal glycaemic control and control of blood pressure are of paramount importance in patients with CKD as in the normal population. Though CVDs are rampant in patients with CKD, there is scarce evidence on the optimal management strategy of this subgroup. This is because almost all the major trials have excluded patients with renal dysfunction. Charytan and Kuntz reviewed 86 trials with over 400000 patients, of which $80 \%$ of the trials excluded ESRD subjects and baseline renal function was reported in only $7 \%$ of the trials. Moreover, the benefits obtained from standard medical therapy in non-CKD population cannot be extrapolated to the CKD population. This leads to the dilemma being faced while treating a patient with renal dysfunction. Observational studies have also shown that optimal drugs are not used adequately in CKD subjects. This was also seen in the study by 
Berger et $a l^{28}$ on over 1000 patients on dialysis, wherein patients with CKD were not treated with optimal medical therapy though they provided mortality benefit. Probable reason for underuse is the fear of worsening renal dysfunction with ACEi/ARB or the presumed increased risk of bleeding with antiplatelet agents. 'Therapeutic nihilism' for conventional standard therapies could be due to lack of confidence in use of these measures in CKD or due to fear of doing harm in these patients with these measures and this reluctance to use proven therapies in patients with CKD leads to dismal prognosis of patients with CVD. Though, there is paucity of randomised trials so far in this topic, a summary of current evidence is presented.

Antiplatelet therapy forms the cornerstone of therapy for CVD with an unequivocal benefit for low dose aspirin even in patients with CKD in all the observational studies. The meta-analysis by the Antithrombotic Trialists' Collaboration showed that low dose aspirin is as good as standard dose unless stents are in place. The UK Heart and Renal Protection (HARP) study and the Dialysis Outcomes and Practise Patterns Study (DOPPS) revealed no increase in major bleeding episodes independent of the stage of CKD. The general opinion is that there is increase in minor bleeding episodes but uncertain risk of major bleeding episodes. A recent Cochrane collaboration systematic review in patients across all stages of CKD showed that antiplatelet agents reduced the risk of myocardial infarction, but not all-cause mortality, cardiovascular mortality or stroke with increased incidence of bleeding episodes. ${ }^{29}$ The risk of bleeding manifestations is compounded as combination antiplatelet therapy is often advised in ACS. Clopidogrel is recommended in patients with ACS but in patients with CKD it has been shown to be less efficacious. Moreover, post hoc analysis of Clopidogrel for the Reduction of Events During Observation (CREDO) and Clopidogrel in Unstable Angina to Prevent Recurrent Events (CURE) trials failed to show any benefit for clopidogrel in patients with CKD. Ticagrelor has shown mortality benefit in patients with renal dysfunction in the Platelet Inhibition and Patient Outcomes (PLATO) trial. Glycoprotein IIb/IIIa inhibitors (GPIs) are potent inhibitors of platelets and subgroups analyses of major studies (Do Tirofiban and ReoPro Give Similar Efficacy Outcome Trial (TARGET), Platelet Receptor Inhibition in Ischemic Syndrome Management in Patients Limited by Unstable Signs and Symptoms study (PRISM-PLUS), European/Australasian Stroke Prevention in Reversible Ischaemia Trial (ESPRIT), Evaluate the Relative Protection Against Post-PCI Microvascular Dysfunction and Post-PCI Ischemia Among Anti-Platelet and Anti-Thrombotic Agents-Thrombolysis In Myocardial Infarction (PROTECT), Can Rapid Risk Stratification of Unstable Angina Patients Suppress Adverse Outcomes With Early Implementation of the ACC/AHA Guidelines (CRUSADE)) have shown treatment benefit with the risk of bleeding. Dose modifications are needed for GPIs in renal insufficiency and use of calculated doses reduces bleeding complications. Heparin is universally administered for ACS. Unfractionated heparin (UFH) and low molecular weight heparin (LMWH) are the two preparations available. LMWH is presently the standard of care but in those with renal insufficiency its half-life is increased 1.7 times. In the EXTRACT trial, it was shown that every $30 \mathrm{~mL} / \mathrm{min}$ decrease in $\mathrm{CrCl}$, the risk of major and minor bleeding increased by $50 \% .^{30}$ Though UFH is comparatively safer, bleeding risk with UFH also increases in parallel with increasing severity of renal dysfunction.

ACEi or ARBs form the standard medical therapy in patients with normal renal function. Beneficial effect of ACEi/ARBs in renal insufficiency is equivocal with the Fosinopril in Dialysis (FOSIDIAL) trial failing to show any additional benefit in patients on dialysis, while the study by Efrati et $a l^{31}$ showed a $52 \%$ reduction in mortality among dialysis patients. Candesartan was found to reduce cardiovascular mortality in patients with ESRD in the study by Takayashi et al. ${ }^{32}$ In the study by Molnar et $a l^{33}$ in 141413 patients, ACEi/ARB use was associated with greater survival in non-dialysis-dependent CKD. Post hoc analysis of the HOPE study revealed similar beneficial effects with ramipril in mild renal insufficiency. However, the Cochrane review in early non-diabetic CKD (stages 1-3) failed to show any benefit in cardiovascular events or all-cause mortality with $\mathrm{ACEi}^{34}$ Unlike ACEi/ARBs, $\beta$ blockers do not cause renal dysfunction but the differential effects of different agents in varying range of renal function has been a subject of concern. Carvedilol has been shown to reduce mortality and risk of cardiovascular events in patients with CKD. In the meta-analysis by Badve et $a l,{ }^{35}$ treatment with $\beta$ blockers improved all-cause mortality in patients with CKD and chronic systolic heart failure across all stages of non-dialysis-dependent patients with CKD.

Use of statins in patients on dialysis was brought to disrepute following the 4D and A Study to Evaluate the Use of Rosuvastatin in Subjects on Regular Hemodialysis: An Assessment of Survival and Cardiovascular Events (AURORA) study. In the Study of Heart and Renal Protection (SHARP) trial, where ezetimibe was used in addition to statins and included patients in all stages of CKD with $32 \%$ on maintenance dialysis, an average reduction of $0.85 \mathrm{mmol} / \mathrm{L}$ in low density lipoprotein (LDL) concentration yielded a significant $17 \%$ reduction in major atherosclerotic events. The Kidney Disease: Improving Global Outcomes (KDIGO) 2013 guidelines recommend statin or statin/ezetimibe treatment in adults aged $\geq 50$ years with eGFR $<60 \mathrm{~mL} / \mathrm{min}$ per $1.73 \mathrm{~m}^{2}$ but not treated with chronic dialysis and in those between 18 and 49 years statin therapy is recommended if there are other risk factors for CVD. Moreover, they do not recommend initiating statins in patients on dialysis but suggest continuation of statin therapy if patient is already receiving it. This guideline differs from the ACC/AHA guidelines in the definition of atherosclerotic CVD (ACC/AHA has a broader definition), in dealing with patients over 75 years, and using a higher risk level of CVD (10-year risk of $10 \%$ vs $7.5 \%$ in ACC/AHA) in deciding statin therapy. ${ }^{36}$ Whether there is an eGFR limit, the so called 'point of no return' beyond which statins lose their benefit is uncertain and physicians should decide on statin therapy on individual merit.

Management of anaemia is an integral part of CKD care and anaemia per se adds to the cardiovascular burden. Thereby it is logical to think that appropriate treatment of anaemia would have cardiovascular benefits. The target haemoglobin for therapy has been a subject of study and with the results of the Correction of Hemoglobin and Outcomes in Renal Insufficiency (CHOIR), Cardiovascular Reduction Early Anemia Treatment Epoetin $\beta$ (CREATE) and Trial to Reduce Cardiovascular Events With Aranesp Therapy (TREAT) studies, KDIGO suggests that erythropoietin therapy should not be used to maintain haemoglobin concentration above $11.5 \mathrm{~g} / \mathrm{dL}$. Risk of hypertension, congestive heart failure and stroke increases as the haemoglobin crosses $12 \mathrm{~g} / \mathrm{dL}$. For optimal cardiovascular and overall benefit, therapy should be titrated to achieve target haemoglobin between 10 and $12 \mathrm{~g} / \mathrm{dL}$.

The only randomised controlled trial (RCT) performed to date comparing medical and revascularisation therapies was in 1992 by Manske et al with 26 patients with CKD and insulindependent diabetes with a 2-year follow-up. Though medical 
therapy was suboptimal with only calcium channel blockers and aspirin, it showed significant benefit with revascularisation therapy. ${ }^{37}$ The presence of small diffusely diseased vessels coupled with vascular calcification in patients with CKD was responsible for high failure rates with balloon angioplasty in the prestent era. Surgical reperfusion by coronary artery bypass grafting (CABG) was considered the gold standard due to restenosis of bare metal stents (BMS) used earlier. The Acute Coronary Syndrome Israeli Survey (ACSIS) that compared different reperfusion strategies in patients with serum creatinine $>1.5 \mathrm{mg} \%$ found 30 -day mortality was $8.3 \%$ in the thrombolysis group compared to $40 \%$ and $29.7 \%$ in the primary PCI and no reperfusion groups respectively. ${ }^{38}$ In one another study treatment with drug eluting stents (DES) compared with BMS resulted in lower angiographic restenosis rates $(2.1 \%$ vs $20.5 \%)$ though there was no mortality benefit at 1 year. In the Global Registry of Acute Coronary Events (GRACE) study, among patients with moderate renal dysfunction, PCI therapy had significant lower mortality at 6 months compared with fibrinolysis. Patients with ESRD have a greater percentage of calcified lesions and greater residual stenosis after the procedure. With the advent of DES, PCI therapy is back in vogue due to lesser risk for restenosis and reduced frequency of repeat procedures. Hobbach et $a l .{ }^{39}$ studied 352 patients with CKD with ST segment elevation myocardial infarction (STEMI) and showed that 30-day and 6-month mortality were reduced from $22 \%$ to $4 \%(\mathrm{p}<0.03)$ and from $25 \%$ to $7 \%(\mathrm{p}<0.05)$ among those who underwent PCI during hospitalisation. With regard to nonST segment elevation myocardial infarction (NSTEMI), early invasive therapy appears to be less beneficial with worser outcomes in patients on dialysis. Data from Korea Acute Myocardial Infarction Registry (KAMIR) and Swedish WebSystem for Enhancement and Development of Evidence-Based Care in Heart Disease Evaluated According to Recommended Therapies (SWEDEHEART) study reiterate this fact. Perioperative death rates following CABG are threefold higher than in the general population. Nevertheless, the reduced necessity for repeat procedures with better long-term prognosis made CABG a favourable mode of therapy. Better outcomes have been achieved with mammary artery grafts than patients who received venous grafts. A recent meta-analysis with 28 studies and 38740 patients concluded that CABG had higher risk of shortterm mortality but lower risks of long-term all-cause mortality, cardiac mortality and late myocardial infarction compared with PCI. ${ }^{40}$

The ongoing International Study of Comparative Health Effectiveness with Medical and Invasive Approaches (ISCHEMIA trial: ClinicalTrials.gov Identifier: NCT01985360) which compares an initial invasive strategy of optimal revascularisation, in addition to optimal medical therapy in patients with stable ischaemic heart disease with a conservative strategy of medical therapy alone also has an ancillary arm of patients with CKD with eGFR $<30 \mathrm{~mL} / \mathrm{min}$ and is likely to provide new insight to the ideal management of these patients.

\section{CONCLUSION}

CKD is an independent risk factor for CVD and majority of patients expire due to CVD than progress to ESRD. This risk worsens as the severity of renal dysfunction worsens. Identification of patients with early CKD is crucial as prevention works better than cure. Apart from the traditional risk factors, novel risk factors peculiar to CKD results in early and rapid progression of CVD. An array of invasive and non-invasive tests is available for diagnosis of CVD but the utility of each of them in patients with CKD is still uncertain. Treatment benefits with reperfusion therapy extend to patients with renal dysfunction but the degree of protection and risks involved needs further evaluation. Most of the recommendations are based on singlecentre data or post hoc analyses. Further randomised control trials are warranted to assess the various modalities for evaluation and management of CVDs in CKD.

Contributors SM conceptualised and reviewed the text, YKC and AKS researched the articles and wrote the manuscript.

Competing interests None declared.

Provenance and peer review Commissioned; externally peer reviewed.

\section{REFERENCES}

1 Briasoulis A, Bakris GL. Chronic kidney disease as a coronary artery disease risk equivalent. Curr Cardiol Rep 2013;15:340.

2 Braam B, Joles JA, Danishwar AH, et al. Cardiorenal syndrome-current understanding and future perspectives. Nat Rev Nephrol 2014;10:48-55.

3 Anavekar NS, Pfeffer MA. Cardiovascular risk in chronic kidney disease. Kidney Int 2004;66:S11-15.

4 Herzog CA, Asinger RW, Berger AK, et al. Cardiovascular disease in chronic kidney disease. A clinical update from Kidney Disease: Improving Global Outcomes (KDIGO). Kidney Int 2011;80:572-86.

5 Go AS, Chertow GM, Fan D, et al. Chronic kidney disease and the risks of death, cardiovascular events, and hospitalization. N Engl J Med 2004;351:1296-305.

6 van der Velde M, Matsushita K, Coresh J, et al. Lower estimated glomerular filtration rate and higher albuminuria are associated with all-cause and cardiovascular mortality. A collaborative meta-analysis of high-risk population cohorts. Kidney Int 2011;79:1341-52.

7 Manjunath G, Tighiouart $\mathrm{H}$, Coresh J, et al. Level of kidney function as a risk factor for cardiovascular outcomes in the elderly. Kidney Int 2003;63:1121-9.

8 Irie $F$, Iso $H$, Sairenchi $T$, et al. The relationships of proteinuria, serum creatinine, glomerular filtration rate with cardiovascular disease mortality in Japanese general population. Kidney Int 2006;69:1264-71.

9 Rahman M, Xie D, Feldman HI, et al. Association between chronic kidney disease progression and cardiovascular disease: results from the CRIC Study. Am I Nephrol 2014;40:399-407.

10 Elsayed EF, Tighiouart $\mathrm{H}$, Griffith J, et al. Cardiovascular disease and subsequent kidney disease. Arch Intern Med 2007;167:1130-6.

11 Gansevoort RT, Correa-Rotter R, Hemmelgarn BR, et al. Chronic kidney disease and cardiovascular risk: epidemiology, mechanisms, and prevention. Lancet 2013;382:339-52.

12 Kendrick J, Chonchol MB. Nontraditional risk factors for cardiovascular disease in patients with chronic kidney disease. Nat Clin Pract Nephrol 2008;4:672-81.

13 Weiner DE, Tighiouart $H$, Vlagopoulos PT, et al. Effects of anemia and left ventricular hypertrophy on cardiovascular disease in patients with chronic kidney disease. J Am Soc Nephrol 2005;16:1803-10.

14 Negri AL. Fibroblast growth factor 23: associations with cardiovascular disease and mortality in chronic kidney disease. Int Urol Nephrol 2014;46:9-17.

15 Gutiérrez OM, Mannstadt M, Isakova T, et al. Fibroblast growth factor 23 and mortality among patients undergoing hemodialysis. N Engl J Med 2008;359:584-92.

16 Cai Q, Mukku VK, Ahmad M. Coronary artery disease in patients with chronic kidney disease: a clinical update. Curr Cardiol Rev 2013;9:331-9.

17 Sharma R, Pellerin D, Gaze DC, et al. Dobutamine stress echocardiography and the resting but not exercise electrocardiograph predict severe coronary artery disease in renal transplant candidates. Nephrol Dial Transplant 2005;20:2207-14.

18 Patel AD, Abo-Auda WS, Davis JM, et al. Prognostic value of myocardial perfusion imaging in predicting outcome after renal transplantation. Am J Cardiol 2003:92:146-51.

19 De Lima JJG, Sabbaga E, Vieira MLC, et al. Coronary angiography is the best predictor of events in renal transplant candidates compared with noninvasive testing. Hypertens 2003;42:263-8.

20 Reis G, Motta MS, Barbosa MM, et al. Dobutamine stress echocardiography for noninvasive assessment and risk stratification of patients with rheumatic mitral stenosis. J Am Coll Cardiol 2004;43:393-401.

21 Vandenberg BF, Rossen JD, Grover-McKay M, et al. Evaluation of diabetic patient for renal and pancreas transplantation: noninvasive screening for coronary artery disease using radionuclide methods. Transplantation 1996:62:1230-5.

22 Rabbat CG, Treleaven DJ, Russell JD, et al. Prognostic value of myocardial perfusion studies in patients with end-stage renal disease assessed for kidney or kidney-pancreas transplantation: a meta-analysis. J Am Soc Nephrol 2003:14:431-9.

23 Wang LW, Masson P, Turner RM, et al. Prognostic value of cardiac tests in potential kidney transplant recipients: a systematic review. Transplantation 2015;99:731-45. 
24 Lentine KL, Costa SP, Weir MR, et al. Cardiac disease evaluation and management among kidney and liver transplantation candidates: a scientific statement from the American Heart Association and the American College of Cardiology Foundation: endorsed by the American Society of Transplant Surgeons, American Society of Transplantation, and National Kidney Foundation. Circulation 2012;126: 617-63.

25 Freda BJ, Tang WHW, Van Lente $F$, et al. Cardiac troponins in renal insufficiency: review and clinical implications. J Am Coll Cardiol 2002;40:2065-71.

26 Stacy SR, Suarez-Cuervo C, Berger Z, et al. Role of troponin in patients with chronic kidney disease and suspected acute coronary syndrome: a systematic review. Ann Intern Med 2014;161:502-12.

27 Wang AY-M, Lai K-N. Use of cardiac biomarkers in end-stage renal disease. J Am Soc Nephrol 2008;19:1643-52.

28 Berger AK, Duval S, Krumholz HM. Aspirin, beta-blocker, and angiotensin-converting enzyme inhibitor therapy in patients with end-stage renal disease and an acute myocardial infarction. J Am Coll Cardiol 2003;42:201-8.

29 Palmer SC, Di Micco L, Razavian M, et al. Antiplatelet agents for chronic kidney disease. Cochrane Database Syst Rev 2013;(2):CD008834.

30 Fox KAA, Antman EM, Montalescot $G$, et al. The impact of renal dysfunction on outcomes in the ExTRACT-TIMI 25 trial. J Am Coll Cardiol 2007;49:2249-55.

31 Efrati S, Zaidenstein R, Dishy V, et al. ACE inhibitors and survival of hemodialysis patients. Am J Kidney Dis 2002;40:1023-9.

32 Takahashi A, Takase H, Toriyama T, et al. Candesartan, an angiotensin II type-1 receptor blocker, reduces cardiovascular events in patients on chronic haemodialysis -a randomized study. Nephrol Dial Transplant 2006;21:2507-12.
33 Molnar MZ, Kalantar-Zadeh K, Lott EH, et al. Angiotensin-converting enzyme inhibitor, angiotensin receptor blocker use, and mortality in patients with chronic kidney disease. J Am Coll Cardiol 2014;63:650-8.

34 Sharma P, Blackburn RC, Parke CL, et al. Angiotensin-converting enzyme inhibitors and angiotensin receptor blockers for adults with early (stage 1 to 3 ) non-diabetic chronic kidney disease. Cochrane Database Syst Rev 2011;(10): CD007751.

35 Badve SV, Roberts MA, Hawley CM, et al. Effects of beta-adrenergic antagonists in patients with chronic kidney disease: a systematic review and meta-analysis. J Am Coll Cardiol 2011;58:1152-61.

36 Stone NJ, Robinson JG, Lichtenstein AH, et al. 2013 ACC/AHA guideline on the treatment of blood cholesterol to reduce atherosclerotic cardiovascular risk in adults: a report of the American College of Cardiology/American Heart Association Task Force on practice guidelines. J Am Coll Cardiol 2014;63:2889-934.

37 Manske CL, Wang Y, Rector $\mathrm{T}$, et al. Coronary revascularisation in insulin-dependent diabetic patients with chronic renal failure. Lancet 1992;340:998-1002.

38 Dragu R, Behar S, Sandach A, et al. Should primary percutaneous coronary intervention be the preferred method of reperfusion therapy for patients with renal failure and ST-elevation acute myocardial infarction? Am J Cardiol 2006;97:1142-5.

39 Hobbach HP, Gibson CM, Giugliano RP, et al. The prognostic value of serum creatinine on admission in fibrinolytic-eligible patients with acute myocardial infarction. J Thromb Thrombolysis 2003;16:167-74.

40 Chen YY, Wang JF, Zhang YJ, et al. Optimal strategy of coronary revascularization in chronic kidney disease patients: a meta-analysis. Eur J Intern Med 2013;24:354-61. 